\title{
Análise do Efeito dos Ventos em um Modelo Populacional Difusivo com Abate Impulsivo via Transformada Integral Generalizada
}

\author{
Maiquison dos Santos Friguis ${ }^{1}$ \\ Instituto Federal Fluminense \\ Antônio José da Silva Neto ${ }^{2}$ \\ Universidade do Estado do Rio de Janeiro \\ Diego Campos Knupp ${ }^{3}$ \\ Universidade do Estado do Rio de Janeiro
}

Resumo. Neste trabalho é realizado o estudo da dinâmica de evolução de uma população difusiva com taxas de natalidade e mortalidade logísticas na presença de armadilhas locais considerando o efeito do vento. O problema tratado é resolvido com a Técnica de Transformada Integral Generalizada. A modelagem do vento se mostra importante uma vez que esse efeito pode provocar mudanças significativas na dinâmica da população estudada.

Palavras-chave. População Difusiva, Vento Advectivo, Abate Impulsivo, Transformada Integral.

\section{Introdução}

A modelagem matemática da evolução populacional é assunto abordado em diversos trabalhos presentes na literatura $[5-7,12]$. A dinâmica de populações é um assunto extremamente importante, pois existem muitas doenças que podem ser transmitidas por vetores [3]. No Brasil os principais vetores de doenças são os mosquitos, responsáveis pela transmissão de diversos vírus [9]. Esse tipo de população está sujeito a condições climáticas [11] além disso, os mosquitos ainda podem ser afetados por massas de ar que podem provocar uma maior dispersão dessa população [8]. Modelos que descrevem essas populações podem ser formulados pela equação da difusão-advecção considerando mecanismos de controle e taxas de natalidade e mortalidade logísticas $[4,10]$. Considerando que as equações que governam este problema são tipicamente não-lineares, optou-se pela Técnica da Transformada Integral Generalizada [1] para a solução do problema direto. Esta escolha possibilitou ainda a escolha de uma solução de baixa ordem, reduzindo drasticamente o custo computacional.

\footnotetext{
${ }^{1}$ maiquison.friguis@iff.edu.br

2ajsneto@iprj.uerj.br

${ }^{3}$ diegoknupp@iprj.uerj.br
} 


\section{Formulação Matemática e Solução do Problema Direto}

Considere que uma população com densidade $u$ localizada em um domínio unidimensional é governada pela lei de Fick com coeficiente de dispersão $D$. Considere também que ventos advectivos com velocidade $\nu$ também podem resultar em um movimento de massas populacionais [8]. Considere ainda que o atraso no tempo de reprodução é $\tau$, a taxa de morte prematura é dada por $\mu$ e a taxas de natalidade e mortalidade são dadas, respectivamente, por $b(u)$ e $d(u)$. A equação que governa este fenômeno é dada por [12]:

$$
\frac{\partial u(x, t)}{\partial t}=D \frac{\partial}{\partial x}\left(\frac{\partial u(x, t)}{\partial x}\right)+e^{-\mu \tau} b(u)-d(u)-\nu \frac{\partial u(x, t)}{\partial x}-\sum_{j=1}^{N_{c s}} B_{j} u\left(x_{j}, t\right) \delta\left(x-x_{j}\right)
$$

para $0<x<L$ e $t>0$, onde $x_{j}$ representa a localização de cada armadilha com intensidade $B_{j}$ com número total dado por $N_{c s}, \delta\left(x-x_{j}\right)$ é a função Delta de Dirac e a condição inicial é dada por:

$$
u(x, t)=u_{0}(x, t), \quad-\tau \leq t \leq 0
$$

juntamente com as condições de contorno, dadas por:

$$
\left.\frac{\partial u}{\partial x}\right|_{x=0}=0,\left.\quad \frac{\partial u}{\partial x}\right|_{x=L}=0
$$

As taxas logísticas de nascimento e morte, $b(u)$ e $d(u)$, são:

$$
b(u)=\frac{u(x, t-\tau)}{K+C u(x, t-\tau)}, \quad d(u)=\frac{P u^{2}(x, t)}{K+C u(x, t-\tau)}
$$

onde $P, K$ e $C$ são parâmetros do modelo de crescimento logístico.

Seguindo o formalismo da Técnica de Transformada Integral Generalizada [1], pode-se definir o seguinte par Transformada-Inversa:

$$
\begin{aligned}
& \text { Transformada: } \bar{u}_{i}(t)=\int_{0}^{L} u(x, t) \widetilde{\psi}_{i}(x) d x \\
& \text { Inversa: } \quad u(x, t)=\sum_{i=1}^{\infty} \widetilde{\psi}_{i}(x) \bar{u}_{i}(t)
\end{aligned}
$$

onde as autofunções normalizadas $\widetilde{\psi}_{i}(x)$ são encontradas pela normalização de autofunções geradas pela solução do problema de autovalor diferencial de Sturm-Liouville, obtido pela aplicação da técnica de separação de variáveis à versão homogênea linear do problema original [1].

Com a aplicação do operador $\int_{0}^{L}(.) \widetilde{\psi}_{i}(x) d x$ ao problema original, o seguinte sistema transformado é obtido:

$$
\frac{d \bar{u}_{i}(t)}{d t}+\lambda_{i}^{2} \bar{u}_{i}(t)=\bar{g}_{i}(t, \mathbf{u})
$$

onde $\lambda_{i}$ são os autovalores associados às autofunções $\widetilde{\psi}_{i}(x)$, e:

$$
\mathbf{u}=\left(\bar{u}_{1}, \bar{u}_{2}, \ldots, \bar{u}_{N}\right)
$$




$$
\bar{g}_{i}(t, \mathbf{u})=\bar{g}_{i, 1}(t, \mathbf{u})+\bar{g}_{i, 2}(t, \mathbf{u})+\bar{g}_{i, 3}(t, \mathbf{u})
$$

onde:

$$
\begin{gathered}
\bar{g}_{i, 1}=\int_{0}^{L} \frac{\widetilde{\psi}_{i}(x) e^{-\mu \tau} \sum_{j=1}^{N} \bar{u}_{j}(t-\tau) \widetilde{\psi}_{j}(x)}{K+C \sum_{j=1}^{N} \bar{u}_{j}(t-\tau) \widetilde{\psi}_{j}(x)} d x \\
\bar{g}_{i, 2}=\int_{0}^{L} \frac{\widetilde{\psi}_{i}(x) P\left(\sum_{j=1}^{N} \bar{u}_{j}(t) \widetilde{\psi}_{j}(x)\right)^{2}}{K+C \sum_{j=1}^{N} \bar{u}_{j}(t-\tau) \widetilde{\psi}_{j}(x)} d x \\
\bar{g}_{i, 3}=\int_{0}^{L} \widetilde{\psi}_{i}(x) \nu \frac{\partial}{\partial x}\left[\sum_{j=1}^{N} \bar{u}_{j}(t) \widetilde{\psi}_{j}(x)\right] d x \\
\bar{g}_{i, 4}=\sum_{k=1}^{N_{c s}} B_{k} \widetilde{\psi}_{i}\left(x_{k}\right)\left(\sum_{j=1}^{N} \bar{u}_{j}(t) \widetilde{\psi}_{j}\left(x_{k}\right)\right)
\end{gathered}
$$

A condição inicial transformada é dada por:

$$
\bar{u}_{i}(t)=\int_{0}^{L} \widetilde{\psi}_{i}(x) u_{0}(x, t) d x, \quad-\tau \leq t \leq 0, \quad i=1, \ldots, N
$$

O sistema infinito acoplado obtido é truncado em uma ordem $N$ e solucionado numericamente através da rotina NDSolve do software Mathematica, com controle automático dos erros absoluto e relativo.

Em [4] verificou-se um alto custo computacional para resolver o problema direto, isso motiva soluções de baixa ordem ou até mesmo implementação de modelos aproximados que permitam algum ganho nesse sentido, neste trabalho optou-se pela seguinte modificação [2]:

$$
\sum_{i=1}^{\infty} \bar{u}_{i}(t) \widetilde{\psi}_{i}(x)=\sum_{i=1}^{N_{R}} \bar{u}_{i}(t) \widetilde{\psi}_{i}(x)
$$

apenas nas Equações (9-11), não alterando o número de equações presentes no sistema acoplado Eq.( 13)

\section{Resultados e Discussões}

Baseando-se no trabalho [12] foram utilizados os valores da Tabela 1 para os parâmetros do modelo e $\nu=0,001$. Na Tabela 2 é apresentado o comportamento da solução do problema direto considerando a presença de armadilhas com intensidade constante $B_{j}, j=$ $1, \ldots, N_{c s}$, posicionadas em $x_{1}=0,2, x_{2}=0,4, x_{3}=0,6$ e $x_{4}=0,8$. Os resultados apresentados foram calculados com $M=150$ sub-regiões na integração semi-analítica.

Na Tabela 2 pode-se observar a convergência da solução considerando diferentes valores para a ordem de truncamento $N$, embora não seja informado na tabela quanto maior o valor de $N$ maior o tempo computacional. Na Figura 1 pode-se ver o efeito da advecção na solução do problema inverso mostrando que quanto maior o valor da advecção menos 
Tabela 1: Parâmetros para solução do problema direto.

\begin{tabular}{ccccccccc}
\hline$D$ & $P$ & $K$ & $C$ & $B$ & $\mu$ & $\tau$ & $L$ & $u_{0}(x, t)$ \\
\hline 0,0001 & 0,005 & 1 & 0,5 & 0,001 & 0,0 & 10 & 1 & 1 \\
\hline
\end{tabular}

Tabela 2: Comportamento de Convergência

\begin{tabular}{|c|c|c|c|c|c|c|}
\hline \multirow[t]{2}{*}{$N$} & \multicolumn{3}{|c|}{$u(x, t=50)$} & \multicolumn{3}{|c|}{$u(x, t=100)$} \\
\hline & $x=0,2$ & $x=0,4$ & $x=$ & $=0,2$ & $x=0,4$ & $x=0,5$ \\
\hline 30 & 9 & & & & & 36 \\
\hline 50 & & & & & & \\
\hline 70 & 1 & & & & & \\
\hline 90 & 9 & & & & & 31 \\
\hline 110 & 0,726225 & 0,713376 & 0,874026 & 0,659482 & 0,603913 & 0,737193 \\
\hline
\end{tabular}

u
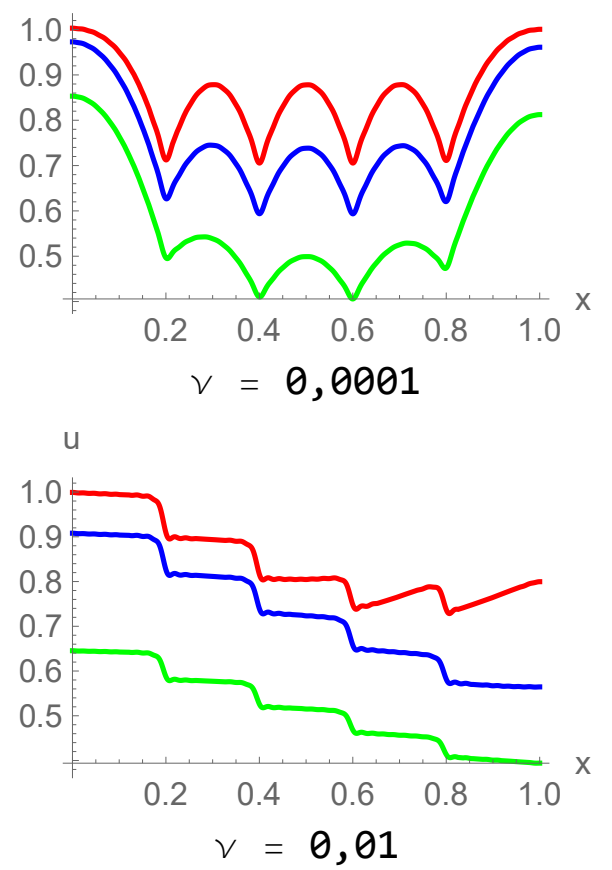

u
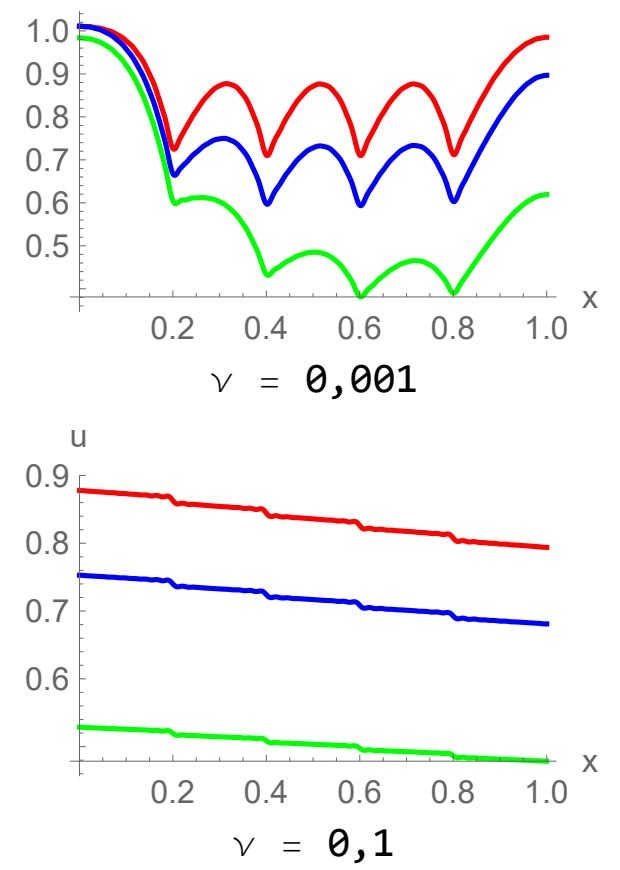

Figura 1: Efeito da Advecção para diferentes tempos. Vermelho $t=50$, azul $t=100$ e verde $t=250$. 


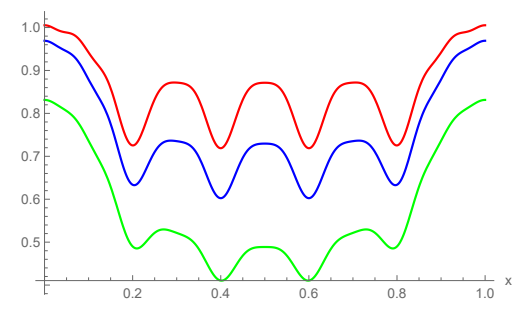

(a) Solução com Modelo Reduzido

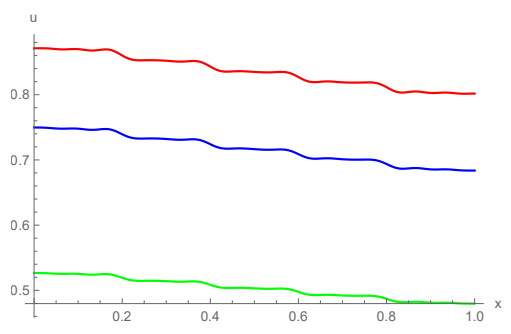

(b) Solução Sem Modelo Reduzido

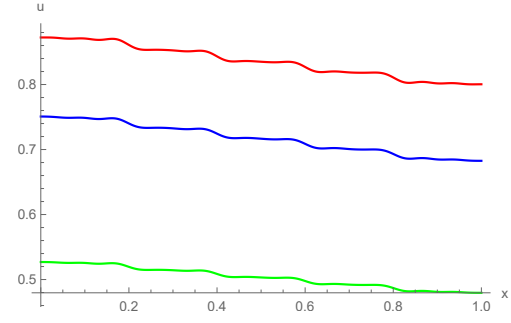

(c) Redução Apenas nas Taxas de Natalidade e Mortalidade Eqs.(910)

Figura 2: Comparação entre modelos

o efeito das armadilhas impulsivas é percebido fazendo com que a população se disperse sem ser atingida.

Na Figura 2a pode-se ver o perfil da solução considerando o modelo reduzido e a solução considerando o modelo completo Figura 2b, pode-se observar que o efeito da advecção não foi recuperado quando utilizou-se o modelo reduzido, porém quando é feita uma redução apenas nos termos responsáveis pelas taxas de natalidade e mortalidade logísticas esse efeito não é suprimido Figura 2c, isso provavelmente deve-se a baixa contribuição que os termos de crescimento fornecem a solução do problema direto quando considerado esse conjunto de parâmetros, porém esse efeito não foi investigado nesse trabalho ficando como sugestão para trabalhos futuros.

\section{Conclusões}

O estudo da dinâmica populacional é um assunto de muita relevância, especialmente quando trata do comportamento de vetores de doenças. Um número muito grande de doenças são transmitidas por vetores de pequeno porte como mosquitos. Na dispersão de uma população de mosquitos o vento pode ser um fator relevante na dinâmica dessa população, neste trabalho utilizou-se um termo advectivo para modelar esse fenômeno e ver seus efeitos para uma população com taxas de natalidade e mortalidade logísticas sujeitas a um mecanismo de controle por meio de abate impulsivo. A solução dessa equação foi implementada considerando o formalismo da técnica de Transformada Integral Generalizada. Notou-se que para maiores valores da velocidade do vento os efeitos das armadilhas 
locais são quase que completamente anulados.

\section{Agradecimentos}

Os autores agradecem ao Instituto Federal Fluminense campus Cabo Frio e as agências FAPERJ, CNPQ E CAPES (Código de financiamento 001) pelo suporte fornecido.

\section{Referências}

[1] R. M. Cotta. Integral Transforms in Computational Heat and Fluid Flow. CRC Press, 1993

[2] M. S. Friguis, D. C. Knupp, L. A. S. Abreu, A. J. da Silva Neto, A. J. Solução de um Problema Inverso em Dinâmica Populacional através de Inferência Bayesiana e os Métodos Luus-Jaakola e Gauss-Newton. Proceeding Series of the Brazilian Society of Computational and Applied Mathematics, 2017.

[3] D. J. Gubler. Resurgent vector-borne diseases as a global health problem. Emerging infectious diseases, v. 4(3), p. 442, 1998.

[4] D. C. Knupp, W. F. Sacco and A. J. Silva Neto. Direct and inverse analysis of diffusive logistic population evolution with time delay and impulsive culling via integral transforms and hybrid optimization, Applied Mathematics and Computation, Elsevier, v. 250, p. $105-120,2015$

[5] A. Okubo and Levin, S.A., Diffusion and ecological problems: modern perspectives. Springer Science \& Business Media, 2001

[6] R. Simons and S.Gourley. Extinction criteria in stage-structured population models with impulsive culling. SIAM Journal on Applied Mathematics, v. 66, n. 6, p. 18531870,2006

[7] J. Skellam. Random dispersal in theoretical populations. Biometrika, v. 38, n. 1/2, p. $196-218,1951$.

[8] L.T Takahashi, N. A Maidana, W. C Ferreira, P. Pulino, and H.M Yang. Mathematical models for the Aedes aegypti dispersal dynamics: travelling waves by wing and wind. Bulletin of mathematical Biology, v. 67(3), p. 509-528, 2005

[9] P.L. Tauil. Perspectivas de controle de doenças transmitidas por vetores no Brasil. Journal of the Brazilian Society of Tropical Medicine, v. 39(3), p. 275-7, 2006.

[10] C. Tian and S. Ruan. On an advection-reaction-diffusion competition system with double free boundaries modeling invasion and competition of Aedes Albopictus and Aedes Aegypti mosquitoes. Journal of Differential Equations, v. 265(9), p. 4016-4051, 2018. 
[11] A. A. Weber, and C.A. Wollmann. A influência climática na proliferação do mosquito Aedes Aegypti em Santa Maria-RS, em 2012. Ciência e Natura, v.38(3) , 2016.

[12] R. E. White. Populations with impulsive culling: control and identification, International Journal of Computer Mathematics, Taylor \& Francis, v. 86, p. 2143-2164, 2009 